OPEN ACCESS

Edited by:

Jan Potempa,

University of Louisville, USA

Reviewed by:

Robert J. C. McLean

Texas State University, USA BN Singh,

National Botanical Research Institute (CSIR), India

*Correspondence: Ermenegilda Parrill erparril@unina.it

these authors have contributed equally to this work.

Received: 07 November 2016 Accepted: 07 February 2017 Published: 23 February 2017

Citation:

Casillo A, Papa R, Ricciardelli A Sannino F, Ziaco M, Tilotta M, Selan L, Marino G, Corsaro MM, Tutino ML,

Artini $M$ and Parrilli E (2017)

Anti-Biofilm Activity of a Long-Chain

Fatty Aldehyde from Antarctic

Pseudoalteromonas haloplanktis TAC125 against Staphylococcus epidermidis Biofilm

Front. Cell. Infect. Microbiol. 7:46 doi: 10.3389/fcimb.2017.00046

\section{Anti-Biofilm Activity of a Long-Chain Fatty Aldehyde from Antarctic Pseudoalteromonas haloplanktis TAC125 against Staphylococcus epidermidis Biofilm}

\author{
Angela Casillo ${ }^{1 \dagger}$, Rosanna Papa ${ }^{2 \dagger}$, Annarita Ricciardelli ${ }^{1}$, Filomena Sannino ${ }^{1}$, \\ Marcello Ziaco' ${ }^{1}$, Marco Tilotta ${ }^{2}$, Laura Selan ${ }^{2}$, Gennaro Marino ${ }^{1}$, Maria M. Corsaro ${ }^{1}$, \\ Maria L. Tutino ${ }^{1}$, Marco Artini ${ }^{2}$ and Ermenegilda Parrilli ${ }^{1 *}$ \\ ${ }^{1}$ Department of Chemical Sciences, Federico II University, Complesso Universitario Monte Sant'Angelo, Naples, Italy, \\ ${ }^{2}$ Department of Public Health and Infectious Diseases, Sapienza University, Rome, Italy
}

Staphylococcus epidermidis is a harmless human skin colonizer responsible for 20\% of orthopedic device-related infections due to its capability to form biofilm. Nowadays there is an interest in the development of anti-biofilm molecules. Marine bacteria represent a still underexploited source of biodiversity able to synthesize a broad range of bioactive compounds, including anti-biofilm molecules. Previous results have demonstrated that the culture supernatant of Antarctic marine bacterium Pseudoalteromonas haloplanktis TAC125 impairs the formation of S. epidermidis biofilm. Further, evidence supports the hydrophobic nature of the active molecule, which has been suggested to act as a signal molecule. In this paper we describe an efficient activity-guided purification protocol which allowed us to purify this anti-biofilm molecule and structurally characterize it by NMR and mass spectrometry analyses. Our results demonstrate that the anti-biofilm molecule is pentadecanal, a long-chain fatty aldehyde, whose anti-S. epidermidis biofilm activity has been assessed using both static and dynamic biofilm assays. The specificity of its action on S. epidermidis biofilm has been demonstrated by testing chemical analogs of pentadecanal differing either in the length of the aliphatic chain or in their functional group properties. Further, indications of the mode of action of pentadecanal have been collected by studying the bioluminescence of a Vibrio harveyi reporter strain for the detection of autoinducer Al-2 like activities. The data collected suggest that pentadecanal acts as an Al-2 signal. Moreover, the aldehyde metabolic role and synthesis in the Antarctic source strain has been investigated. To the best of our knowledge, this is the first report on the identification of an anti-biofilm molecule form from cold-adapted bacteria and on the action of a long-chain fatty aldehyde acting as an anti-biofilm molecule against S. epidermidis.

Keywords: anti-biofilm, Staphylococcus Pseudoalteromonas haloplanktis TAC125 epidermidis, long fatty acid aldehyde, quorum sensing, 


\section{INTRODUCTION}

Staphylococcus epidermidis (S. epidermidis) is now being recognized as an important opportunistic pathogen that can cause significant problems when breaching the epithelial barrier, especially during the biofilm-associated infection of indwelling medical devices (Otto, 2008; Arciola et al., 2012). Most diseases caused by $S$. epidermidis are of a chronic character and occur as device-related infections (such as intravascular catheter or prosthetic joint infections) and/or their complications (Dohar et al., 2009; Artini et al., 2013). Implantations of medical devices are steadily increasing and thus heightening the relevance of $S$. epidermidis as a human pathogen. The ability of S. epidermidis to adhere on both eukaryotic cells and abiotic surfaces and to form biofilm is an essential virulence factor that contributes to the chronicization of infections particularly difficult to eradicate. Biofilms are sticky, surface-attached agglomerations of bacteria that are embedded in an extracellular matrix and provide protection for single cells from antibiotics and mechanisms of host defense (Epstein et al., 2012).

S. epidermidis infections are estimated to reach 250,000 cases per year in the USA with a mortality rate of up to $25 \%$. The interest in the development of innovative approaches for the prevention and treatment of staphylococcal adhesion and biofilm formation capabilities has therefore increased. A viable approach should target the staphylococcal adhesive properties without affecting the bacterial viability in order to avoid the rapid appearance of escape mutants (Papa et al., 2015).

From another point of view, biofilm could be considered as a source of novel drugs. Indeed, the specific environmental conditions prevailing within biofilms may induce a profound genetic and metabolic rewiring of biofilm-dwelling bacteria and therefore may allow the production of metabolites different from those obtained in the planktonic condition. Indeed, many bacterial biofilms secrete molecules such as quorum sensing signals (Ni et al., 2009), surfactants (Kiran et al., 2010), enzymes (Kaplan, 2010), and polysaccharides (Valle et al., 2006; Qin et al., 2009) that act by regulating the biofilm architecture or mediating the release of cells from biofilms during the dispersal stage of the biofilm life cycle (Kaplan, 2010). Furthermore, the production of extracellular molecules that degrade adhesive components in the biofilm matrix is a basic mechanism used in the biological competition between phylogenetically different bacteria (Kaplan, 2010).

Starting from the idea that the production of an anti-biofilm compound might result from the selective pressure exerted on bacteria living in oligotrophic and extreme environments like Antarctica, cold-adapted marine bacteria have been investigated as a possible source of anti-biofilm molecules. Previous papers (Klein et al., 2011; Papa et al., 2013b, 2015; Parrilli et al., 2015, 2016; Sun et al., 2015) have confirmed that cold adapted bacteria represent an untapped reservoir of biodiversity able to synthesize a broad range of potentially valuable bioactive compounds (Klein et al., 2011), including anti-biofilm molecules (Papa et al., 2013b; Parrilli et al., 2015, 2016).

In particular, the Antarctic bacterium Pseudoalteromonas haloplanktis TAC125 (P. haloplanktis TAC125), when grown with a sessile life-style, proved to be able to secrete an anti-biofilm molecule capable of inhibiting S. epidermidis biofilm formation (Papa et al., 2013b; Parrilli et al., 2015, 2016). This anti-biofilm compound impairs biofilm development and disaggregates the mature biofilm of $S$. epidermidis, even in dynamic conditions, without affecting the bacterial viability, showing that its action is specifically directed against biofilm (Papa et al., 2013b; Parrilli et al., 2015, 2016).

In this research study the anti-biofilm molecule produced by $P$. haloplanktis TAC125 has been purified and characterized. In detail, a $P$. haloplanktis TAC125 biofilm cultivation in automatic bioreactor (Parrilli et al., 2016) has been used to obtain a cellfree supernatant in a sufficient amount to purify and characterize the anti-biofilm molecule. A suitable purification protocol was developed using an activity-guided fractionation strategy. The structure of the purified molecule, obtained by NMR and mass spectrometry, corresponded to pentadecanal, a long chain fatty aldehyde. Several experiments were performed to assess the chemical features responsible for its activity, by testing chemical analogs differing in the length of the aliphatic chain and in their functional group properties. Therefore, the anti-biofilm activity of different long chain alcohols and aldehydes on S. epidermidis biofilm was evaluated. As for the anti-biofilm mode of action, the results reported demonstrated that the long-chain fatty aldehyde works as an AI-2 signal, suggesting that it may interfere with the S. epidermidis quorum sensing system. Moreover, in this paper we have investigated the role of pentadecanal in the metabolism of the source strain, in order to clarify if the molecule regulates biofilm development also in the Antarctic bacterium.

\section{MATERIALS AND METHODS}

\section{Bacterial Strains and Culture Conditions}

Bacterial strains used in this work were: S. epidermidis O-47 isolated from clinical septic arthritis and kindly provided by Prof. Gotz (Heilmann et al., 1996); S. epidermidis RP62A reference strain isolated from infected catheter (ATCC collection no. 35984); P. haloplanktis TAC125 (Médigue et al., 2005) collected in 1992 from seawater near French Antarctic Station Dumont d'Urville. Bacteria were grown in Brain Heart Infusion broth (BHI, Oxoid, UK) and in Tryptic Soy Broth (TSB, Oxoid, UK). Biofilm formation was assessed in static condition while planktonic cultures were performed under vigorous agitation (180 rpm).

Vibrio harveyi BB170 (luxN::Tn5kan) (ATCC ${ }^{\circledR}$ BAA-1117 ${ }^{\mathrm{TM}}$ ) was grown as suggested by suppliers, cultivated in the Autoinducer Bioassay (AB) Medium (Taga, 2005) and incubated aerobically on a rotary shaker at $30^{\circ} \mathrm{C}$ under vigorous agitation (180 rpm).

All strains were maintained at $-80^{\circ} \mathrm{C}$ in cryovials with $15 \%$ of glycerol.

\section{Bioluminescence Assay}

To screen the ability of the compounds to interfere with QS, the test products were serially diluted in the $\mathrm{AB}$ medium using sterile, black, clear-bottom 96-well microtiter plates (Greiner Bio-one 
\#655090) as assay platform. An overnight culture of $V$. harveyi BB170 was diluted 1:100 in fresh $\mathrm{AB}$ medium and incubated at $30^{\circ} \mathrm{C}$ up to a visible increase in basal bioluminescence. 100 $\mu l$ of the diluted culture were dispensed inside each well in the microtiter plate starting from a cellular density of about 0.05 (OD $_{600 \mathrm{~nm}} ; 100 \mu \mathrm{l}$ of opportune dilutions of pentadecanal in $\mathrm{AB}$ medium were added into each well. Luminescence and $(\mathrm{OD})_{600 \mathrm{~nm}}$ were monitored every $15 \mathrm{~min}$ over $18 \mathrm{~h}$ with a high-performance multimode plate reader $\left(\right.$ GloMax $^{\circledR}$ Discover System, Promega) in order to correlate the effects of the products on both the growth and bioluminescence kinetics. Data are reported as light units (LU).

\section{Large Scale Biofilm Cultivation of $P$. haloplanktis TAC125 in Automatic Bioreactor for the Anti-Biofilm Compound/s Production}

P. haloplanktis TAC125 bacterial culture was grown in BHI medium in a Stirred Tank Reactor $3 \mathrm{~L}$ fermenter (Applikon) connected to an ADI-1030 Bio Controller (Applikon) with a working volume of $1 \mathrm{~L}$. The bioreactor was equipped with the standard $\mathrm{pH}-, \mathrm{pO}_{2-}^{-}$, level-, and temperature sensors for the bioprocess monitoring. To allow the biofilm formation, autoclaved solid polystyrene supports were added into the bioreactor (33 supports in $1 \mathrm{~L}$ ). The culture was carried out at $15^{\circ} \mathrm{C}$ for $48 \mathrm{~h}$, or at $4^{\circ} \mathrm{C}$ for $96 \mathrm{~h}$, in aerobic conditions using airflow of $6 \mathrm{~L} / \mathrm{h}$, without stirring. After incubation, supports were removed from the supernatant and the adherent cells were recovered by sonication as previously described (Parrilli et al., 2016). In parallel the supernatant was recovered and further separated from cells by centrifugation at 13,000 rpm, sterilized by filtration through membranes with a pore diameter of $0.22 \mu \mathrm{m}$ and stored at $4^{\circ} \mathrm{C}$ until use.

\section{P. haloplanktis TAC125 Growth in Aerobiosis and Microaerobiosis}

Batch cultivations were performed in a computer-controlled bioreactor (Sixfors System, Infors) equipped with control units for $\mathrm{pH}$ and temperature, an oxygen sensing electrode measuring the solution oxygen pressure, and mechanical stirring (200 $\mathrm{rpm}$ ) at $4^{\circ} \mathrm{C}$. Each fermentation unit, filled with medium and sterilized by autoclaving, was equilibrated at process temperature. In order to allow oxygenation of the medium, the stirring speed was set up at the maximum value to be used during the fermentation, and then sterile air supply was switched on. The system was left to stabilize for at least $30 \mathrm{~min}$ to guarantee the saturation of the medium with air. In these conditions $100 \%$ of measured oxygen pressure was assigned. The zero-point set calibration was performed by saturating the medium with sterile nitrogen gas. Under aerobiosis conditions, measured oxygen pressure was maintained always above $20 \%$ by modifying stirring speed and aeration rate. In microaerobiosis (measured oxygen pressure always below 5\% saturation) air supply was stopped after inoculum. For each strain, the growth kinetics were followed in triplicate in at least two independent experiments.

\section{Molecular Methods and Reagents Suppliers}

Standard methods were employed for DNA manipulation and isolation, amplification by PCR, and DNA sequencing. Restriction enzymes, T4 DNA ligase, alkaline phosphatase, T4 polynucleotide kinase, Klenow fragment, Taq DNA polymerase were supplied from Boehringer-Roche, Amersham-Pharmacia Biotech, Promega, and New England Biolabs. DNA fragment purification was carried out with the QUIAEX II kit from Qiagen $\mathrm{GmbH}$.

\section{Vector pVSb0219 and $P$. haloplanktis TAC125-b0219 Mutant Construction}

PCR was employed to amplify a DNA fragment of PSHAb0219 gene. P. haloplanktis TAC125 genomic DNA was used as PCR template and two primers were designed to amplify a 275 bp-long region of the PHSAb0219 gene and to introduce an EcoRI and a SphI site (Oligo b0219EcoRIfw 5'-CTATGAATTCAAGAAGATATTTACGAGC-3' and Oligo b0219SphIrv 5'-AATACCCGCATGCCGTTGGTGCC-3'). The amplified DNA fragment was digested by EcoRI and SphI, and inserted into the pVS plasmid (Giuliani et al., 2012) corresponding site, thus obtaining the pVSb0219 vector. The vector was mobilized by intergeneric conjugation into $P$. haloplanktis TAC125 cells and insertion mutants were screened on plates at $4{ }^{\circ} \mathrm{C}$ containing carbenicillin $(30 \mu \mathrm{g} / \mathrm{ml})$ as selection agent.

\section{RNA Preparation and RT-PCR}

Total RNA was isolated from $500 \mu \mathrm{l}$ aliquots of $P$. haloplanktis TAC125 cells recovered from both planktonic growth and, after sonication of supports, biofilm growth as reported in literature (Rippa et al., 2012). RNA was reverse transcribed using SuperScript II RNase H- reverse transcriptase (Invitrogen) according to the manufacturer's instructions. PHSAb0219 was reverse transcribed starting from total RNA $(\sim 5 \mu \mathrm{g})$ using as primer-specific oligonucleotide designed on the $3^{\prime}$ region of the gene (RT-PCR-Rev $5^{\prime}$-AATACCCGCATGCCGTTGGTGCC$\left.3^{\prime \prime}\right)$. The reaction mix was denatured at $65^{\circ} \mathrm{C}$ for $5 \mathrm{~min}$ and reverse transcribed at $45^{\circ} \mathrm{C}$ for $50 \mathrm{~min}$. cDNA (275 $\mathrm{bp}$ ), the cDNA was amplified using primers Oligo b0219 EcoRIfw $5^{\prime}$-CTATGAATTCAAGAAGATATTTACGAGC- $3^{\prime}$ and Oligo b0219 SphIrv 5' -AATACCCGCATGCCGTTGGTGCC-3', and Taq polymerase (Promega, Madison, WI, USA) according to the manufacturer's instructions. The reaction mix was amplified (denaturation at $95^{\circ} \mathrm{C}$ for $45 \mathrm{~s}$; annealing at $58^{\circ} \mathrm{C}$ for $45 \mathrm{~s}$; extension at $72^{\circ} \mathrm{C}$ for $1 \mathrm{~min}, 35$ cycles). For each reverse transcriptase amplification, an additional PCR reaction on DNAfree total RNA was performed as control, in order to exclude false positive signals (Figure S3).

\section{Determination of Minimal Inhibitory Concentration (MIC)}

MIC was performed according to the guidelines of Clinical Laboratory Standards Institute. Pentadecanal was added directly from mother stock and solutions were prepared by two-fold 
serial dilutions. A total of 5 concentrations were used within the $1.6-0.1 \mu \mathrm{g} / \mathrm{ml}$ range. Experiments were performed in quadruplicate. The MIC was determined as the lowest concentration at which the observable bacterial growth was inhibited. No inhibition of the bacterial growth was evidenced at testedconcentrations.

\section{BIOFILM FORMATION OF STAPHYLOCOCCI}

\section{Static Biofilm Assay}

The quantification of in vitro biofilm production was based on the method described by Christensen with slight modifications (Artini et al., 2013; Papa et al., 2013a). Briefly, the wells of a sterile 48-well flat-bottomed polystyrene plate were filled with $400 \mu \mathrm{l}$ of BHI or TSB medium. 1/100 Dilution of overnight bacterial cultures was added into each well. The first row contained the untreated bacteria, while each of the remaining rows contained serial dilutions of the supernatant (SN) starting from 1:2. The plates were incubated aerobically for $24 \mathrm{~h}$ at $37^{\circ} \mathrm{C}$.

The biofilm formation was measured using crystal violet staining. After treatment, planktonic cells were gently removed; each well was washed three times with PBS and patted dry with a piece of paper towel in an inverted position. To quantify the biofilm formation, each well was stained with $0.1 \%$ crystal violet and incubated for $15 \mathrm{~min}$ at room temperature, rinsed twice with double-distilled water, and thoroughly dried. The dye bound to adherent cells was solubilized with $20 \%(\mathrm{v} / \mathrm{v})$ glacial acetic acid and $80 \%(\mathrm{v} / \mathrm{v})$ ethanol. After $30 \mathrm{~min}$ of incubation at room temperature, (OD) was measured at $590 \mathrm{~nm}$ to quantify the total biomass of biofilm formed in each well. Each data point is composed of three independent experiments, each performed at least in 3-replicates.

\section{Dynamic Biofilm Assay}

To continuously monitor the biofilm development in dynamic condition, we utilized a BioFlux 2,000 microfluidic system (Fluxion Biosciences Inc., San Francisco, CA), which allows the acquisition of microscopic images over time using the experimental protocol previously set up (Iebba et al., 2014). Each flow channel connects to an input well (inlet) and an output well (outlet) on the plate. To grow the biofilm in the BioFlux system, the channels were first primed. We have filled the outlet with $100 \mu \mathrm{l}$ of sterile distilled water and set the flow at a share setting of 1 dyne $/ \mathrm{cm}^{2}$ for $2 \mathrm{~min}$. Coating with $100 \mu \mathrm{l}$ of $10 \mu \mathrm{g} / \mathrm{ml}$ fibronectin was made for $2 \mathrm{~min}$ at $1 \mathrm{dyne} / \mathrm{cm}^{2}$. The fibronectin binding was performed for $30 \mathrm{~min}$ without flow. After priming, fibronectin was aspirated from the output wells and replaced with $100 \mu \mathrm{l}$ of fresh overnight cultures diluted to an (OD) 600 of 0.8 . The channels were seeded by pumping from the output wells to the input wells at $2.0 \mathrm{dyne} / \mathrm{cm}^{2}$ for $4 \mathrm{sec}$. Bacterial adhesion was performed for $30 \mathrm{~min}$ at $37^{\circ} \mathrm{C}$ without flow. $2.0 \mathrm{~mL}$ of $\mathrm{BHI}$ was added to the input well and pumped at $1 \mathrm{dyne} / \mathrm{cm}^{2}$ for 12 h. We used two inlet wells; in the first well we added only BHI. In the second well we added pentadecanal at a concentration of $120 \mu \mathrm{g} / \mathrm{ml}$. Bright-field images were taken at 40X magnification at 1 -min intervals for a total of 720 time points.

\section{Anti-Biofilm Molecule Purification and Identification}

As reported in the previous paper (Parrilli et al., 2016), P. haloplanktis TAC125 supernatant deriving from sessile growth was dialyzed, and the permeated water (2 $\mathrm{g}$ ) was then purified on a gel-filtration column (Biorad, Biogel P-2) eluted with MilliQ water. The active fractions were collected and fractionated on a $\mathrm{C}_{18}$ reverse phase column (Sigma, $30 \mathrm{~mL}, 40 \times 0.5 \mathrm{~cm}$, fraction volume $4 \mathrm{~mL}$ ), eluted with $\mathrm{CH}_{3} \mathrm{CN}: \mathrm{H}_{2} \mathrm{O}$ ranging from 10 to $95 \%$ of $\mathrm{CH}_{3} \mathrm{CN}$. The fraction resulted to be active (S) was analyzed on a Agilent Technologies gas chromatograph 6850A equipped with a mass selective detector $5973 \mathrm{~N}$ and a Zebron ZB-5 capillary column (Phenomenex, $30 \mathrm{~m} \times 0.25$ mmi.d., flow rate $1 \mathrm{~cm}^{3} / \mathrm{min}$, He as carrier gas), by using the following temperature program: $150^{\circ} \mathrm{C}$ for $3 \mathrm{~min}$, from 150 to $300^{\circ} \mathrm{C}$ at $15^{\circ} \mathrm{C} / \mathrm{min}$, at $300^{\circ} \mathrm{C}$ for $5 \mathrm{~min}$.

\section{NMR Spectroscopy}

NMR spectra were performed by using a Bruker Avance-DRX $600 \mathrm{MHz}$ spectrometer equipped with a cryoprobe. ${ }^{1} \mathrm{H}^{1} \mathrm{H}$ DQFCOSY, ${ }^{1} \mathrm{H}_{-}{ }^{1} \mathrm{H}$ TOCSY, ${ }^{1} \mathrm{H}_{-}{ }^{13} \mathrm{C}$ DEPT-HSQC, and ${ }^{1} \mathrm{H}_{-}{ }^{13} \mathrm{C}$ HMBC experiments were recorded at $298 \mathrm{~K}$ in $\mathrm{CDCl}_{3}$. The mixing time for TOCSY experiment was $100 \mathrm{~ms}$.

\section{Fatty Aldehydes Chemical Synthesis}

C14-C16 aldheydes were purchased from TCI. All the other tested compounds were synthesized starting from the corresponding alcohols (Sigma). 1- heptadecanol (30 mg, 0.12 $\mathrm{mmol}$ ) was charged into a $10 \mathrm{~mL}$ round-bottom flask equipped with a magnetic stir bar. The solid was then dissolved in toluene $(2 \mathrm{~mL})$ at $20^{\circ} \mathrm{C}$, and an aqueous solution of sodium bicarbonate $(0.2 \mathrm{~g}, 2.38 \mathrm{mmol}$ in $2 \mathrm{~mL}$ of deionized water) was prepared and charged into the toluene slurry. Solid iodine (2.0 eq., 60 $\mathrm{mg}, 0.24 \mathrm{mmol}$ ) was then charged to the alcohol followed by solid TEMPO (0.1 eq., $1.87 \mathrm{mg}, 12.0 \mathrm{\mu mol}$; Miller and Hoerrner, 2003). The reaction mixture was then left under stirring for $16 \mathrm{~h}$ at $20^{\circ} \mathrm{C}$. The batch was cooled to $5^{\circ} \mathrm{C}$, diluted with ethyl acetate $(2 \mathrm{~mL})$, and quenched at $5^{\circ} \mathrm{C}$ by adding an aqueous solution of sodium sulfite $10 \%$ ( $312 \mathrm{mg}$ sodium sulfite in $2 \mathrm{~mL}$ of deionized water). The quenched reaction mixture was transferred into a separatory funnel, rinsed with additional ethyl acetate (10 $\mathrm{mL}$ ) and deionized water $(10 \mathrm{~mL})$, and the aqueous layer was cut away. The organic layer was then washed with $10 \mathrm{~mL}$ of saturated aqueous sodium bicarbonate, followed by $10 \mathrm{~mL}$ of brine. The organic layer was then dried over sodium sulfate, filtrated, and concentrated in vacuum to a volume of $4 \mathrm{~mL}$. Then, the solution was completely dried under a stream of argon, to give the aldehyde with a $95 \%$ yield.

The same procedure was then utilized for the alcohols 1octadecanol, 1-nonadecanol, and 1-eicosanol for the obtainment of the corresponding aldehydes.

\section{Statistics and Reproducibility of Results}

The data reported were statistically validated using the Student's $t$-test comparing the mean absorbance of treated and untreated samples. The significance of differences between the mean 
absorbance values was calculated using a two-tailed Student's $t$-test. A $p<0.05$ was considered significant.

\section{Bacterial Viability and Biofilm Thickness Determined by Confocal Laser Scanning Microscopy}

For confocal microscopy biofilms were formed on Nunc ${ }^{\mathrm{TM}}$ Lab-Tek ${ }^{\circledR}$ 8-well Chamber Slides $\left(\mathrm{n}^{\circ}\right.$ 177445; Thermo Scientific, Ottawa, ON, Canada). Briefly, overnight cultures of S. epidermidis O-47 and RP62A grown in BHI were diluted and inoculated into each well of the chamber slide to a cell concentration of about $0.001(\mathrm{OD})_{600 \mathrm{~nm}}$. The bacterial cultures were incubated at $37^{\circ} \mathrm{C}$ for $20 \mathrm{~h}$ in presence of pentadecanal $(100$ $\mu \mathrm{g} / \mathrm{ml}$ ) in order to assess its anti-biofilm activity and its influence on cell viability.

The biofilm cell viability was determined with the FilmTracer ${ }^{\mathrm{TM}}$ LIVE/DEAD ${ }^{\circledR}$ Biofilm Viability Kit (Molecular Probes, Invitrogen, Carlsbad, California) following the manufacturer's instructions. After rinsing with filter-sterilized PBS, each well of the chamber slide were filled with $300 \mu \mathrm{l}$ of working solution of fluorescent stains, containing the SYTO ${ }^{\circledR}$ 9 green fluorescent nucleic acid stain $(10 \mu \mathrm{M})$ and Propidium iodide, the red-fluorescent nucleic acid stain $(60 \mu \mathrm{M})$, and incubated for 20-30 min at room temperature, protected from light. All excess stain was removed by rinsing gently with filter-sterilized PBS.

All microscopic observations and image acquisitions were performed with a confocal laser scanning microscope (CLSM; LSM700-Zeiss, Germany) equipped with an Ar laser (488 nm), and a He-Ne laser $(555 \mathrm{~nm})$. Images were obtained using a 20X/0.8 objective. The excitation/emission maxima for these dyes are $\sim 480 / 500 \mathrm{~nm}$ for SYTO $^{\circledR} 9$ stain and 490/635 nm for propidium iodide. Z-stacks were obtained by driving the microscope to a point just out of focus on both the top and bottom of the biofilms. Images were recorded as a series of.tif files with a file-depth of 16 bits. For each condition, two independent biofilm samples were used.

\section{RESULTS}

\section{Anti-Biofilm Production and Purification}

We have previously (Papa et al., 2013b; Parrilli et al., 2015) demonstrated that the cell-free supernatant of the Antarctic bacterium P. haloplanktis TAC125 inhibits S. epidermidis biofilm formation and that the bacterium produces the active molecule only when it is grown in a sessile condition (Papa et al., 2013b). To obtain a sufficient amount of cell-free supernatant, we adopted a recently described (Parrilli et al., 2016) biofilm cultivation strategy for $P$. haloplanktis TAC125. This consists in a fluidizedbed reactor fermentation in the presence of floating polystyrene supports used to increase the biofilm formation (Parrilli et al., 2016). In this work the Antarctic bacterium has been grown in the presence of floating polystyrene supports in $\mathrm{BHI}$ in a 3 $\mathrm{L}$ tank reactor at $4^{\circ} \mathrm{C}$ without stirring, using an airflow of $6 \mathrm{~L}$ $\mathrm{h}^{-1}$ (see materials and Methods Section). The obtained cell-free supernatant was recovered after $96 \mathrm{~h}$ and separated from the floating polystyrene supports and the cells by a centrifugation at 13,000 rpm. Subsequently the supernatant was sterilized by filtration through membranes with a pore diameter of $0.22 \mu \mathrm{m}$, and then underwent dialysis treatment using a semipermeable membrane with a cut-off of $3.5 \mathrm{KDa}$. The permeate product after lyophilization was fractionated on a Biogel P-2 column and the anti-biofilm activity of each fraction was evaluated (data not shown). The fraction endowed with anti-biofilm activity on $S$. epidermidis $\mathrm{O}-47$ was further purified on a $\mathrm{C}_{18}$ reverse phase column eluted with acetonitrile/water. Several fractions (A-S) were collected, and the anti-biofilm activity of each fraction was tested as reported in Figure 1A. Fraction S, eluted with 95\% acetonitrile, showed the highest inhibitory activity on $S$. epidermidis biofilm. The analysis of the ${ }^{1} \mathrm{H}$ NMR spectrum of this fraction revealed a signal at $\delta 9.77 \mathrm{ppm}$, which immediately suggested the presence of an aldehyde (MOLBASE.COM CAS. No. 2765-11-9). This was believed to be aliphatic, due to the presence of intense signals in the range between $\delta 0.1-2.5 \mathrm{ppm}$ (data not shown). To confirm this hypothesis, and to check the purity of the sample, a GC-MS analysis was performed (Figure 1B). The chromatogram clearly indicated two different compounds, named $\mathbf{A}$ and $\mathbf{B}$, the EI mass spectra of which are reported (Figures 1C,D). Both spectra showed signals at $\mathrm{m} / \mathrm{z} 43$, 57,71 , and 85 , that are typical of a straight chain hydrocarbon backbone, thus confirming the aliphatic character of the two molecules. As this type of fragmentation can be attributed to a broad range of compounds, a research in the NIST library was required. The results indicated with a high score a 2-tridecanone and a pentadecanal for $\mathbf{A}$ and $\mathbf{B}$, respectively. As the inactive fraction $\mathrm{Q}$ mainly contains the ketone whereas fraction $\mathrm{S}$ shows a higher content of the pentadecanal, we concluded that the activity should be assigned to the aldehyde. To exclude the possibility that the mass spectrum of $\mathbf{B}$ could belong to an aldehyde with a short difference in the carbon chain length, we injected into the gas chromatograph commercial $\mathrm{C} 14-\mathrm{C} 16$ aldehydes. The retention time of compound $\mathbf{B}$ proved to correspond to that of pentadecanal.

To confirm the data obtained from GC-MS, the $\mathbf{S}$ fraction was analyzed by 2D-NMR spectroscopy. In particular, two-dimensional ${ }^{1}{ }^{1}-{ }^{1} \mathrm{H} \quad$ DQF-COSY (double quantumfiltered correlation spectroscopy), ${ }^{1} \mathrm{H}_{-}{ }^{1} \mathrm{H}$ TOCSY (total correlation spectroscopy), ${ }^{1} \mathrm{H}_{-}{ }^{13} \mathrm{C}$ DEPT-HSQC (distortionless enhancement by polarization transfer-heteronuclear single quantum coherence), and ${ }^{1} \mathrm{H}_{-}{ }^{13} \mathrm{C}$ HMBC (heteronuclear multiple bond correlation) experiments were performed. The proton at $\delta 9.79 \mathrm{ppm}$, that clearly indicated an aldehyde functional group on the molecule, displayed a correlation with a carbon at $\delta 203.1 \mathrm{ppm}$ (Figure 2A; data not shown). In turn, this carbon showed a long range scalar connectivity with protons at $\delta 2.43 \mathrm{ppm}\left(\mathrm{CH}_{2}, \mathrm{C} 2\right.$; Figure $\left.2 \mathrm{~B}\right)$. Starting from this proton signal, the correlations revealed in the COSY and TOCSY spectra allowed us to attribute all the $\mathrm{CH}_{2}(\mathrm{C} 3-\mathrm{C} 14)$ that constitute the aliphatic chain. The last signal, attributable to the $\mathrm{CH}_{2} \mathrm{C} 14(8 \mathrm{H} / \mathrm{C}$ $1.36 / 22.9$ ), showed a long-range cross-peak, with the methyl group at $\delta \mathrm{H} / \mathrm{C} 0.9 / 13.6 \mathrm{ppm}$ (Figure 2C).

A confirmation of the correct assignment of the pentadecanal structure was obtained by testing the commercial product on 
A

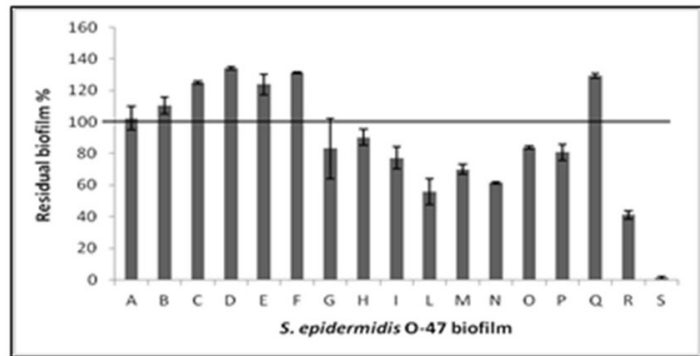

C

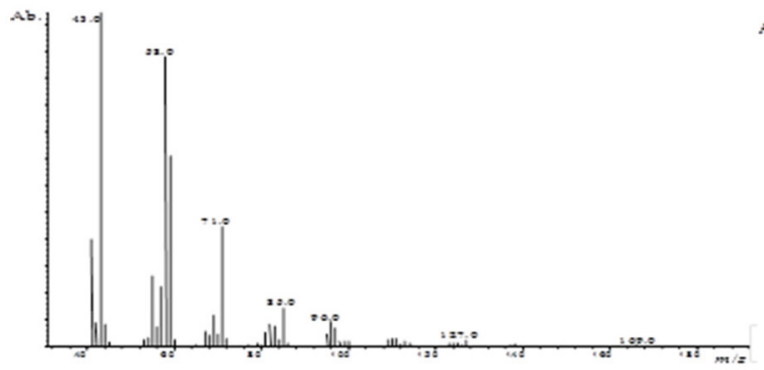

B

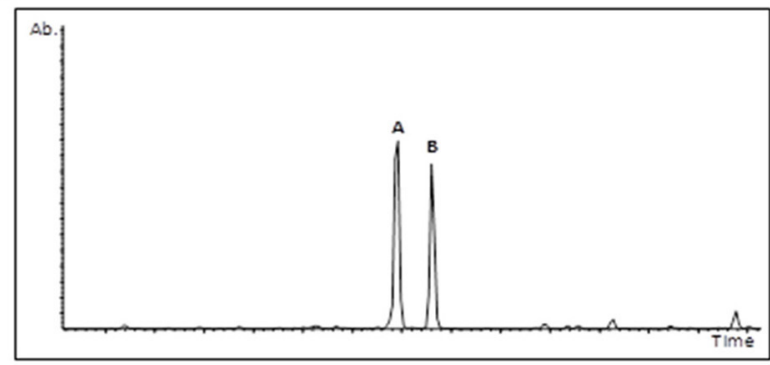

D

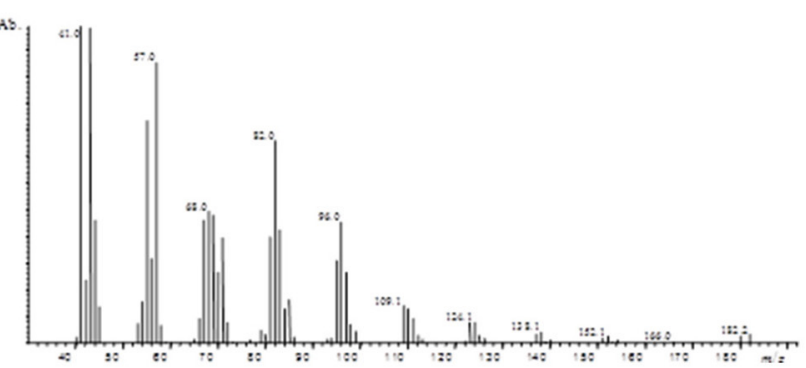

FIGURE 1 | Anti-biofilm assay and identification by GC-MS analysis. (A) The anti-biofilm activity of different fractions obtained from reverse phase $\mathrm{C}_{18}$ column. The fraction S eluted with 95\% acetonitrile showed the highest inhibition activity. (B) GC-MS chromatogram of the fraction S. (C) Mass spectrum of compound A, and (D) compound B.
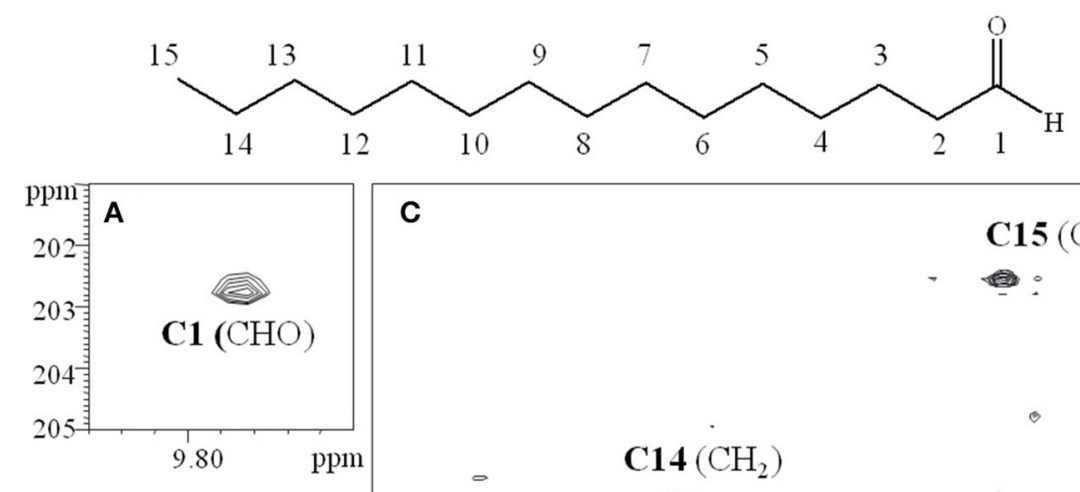

C
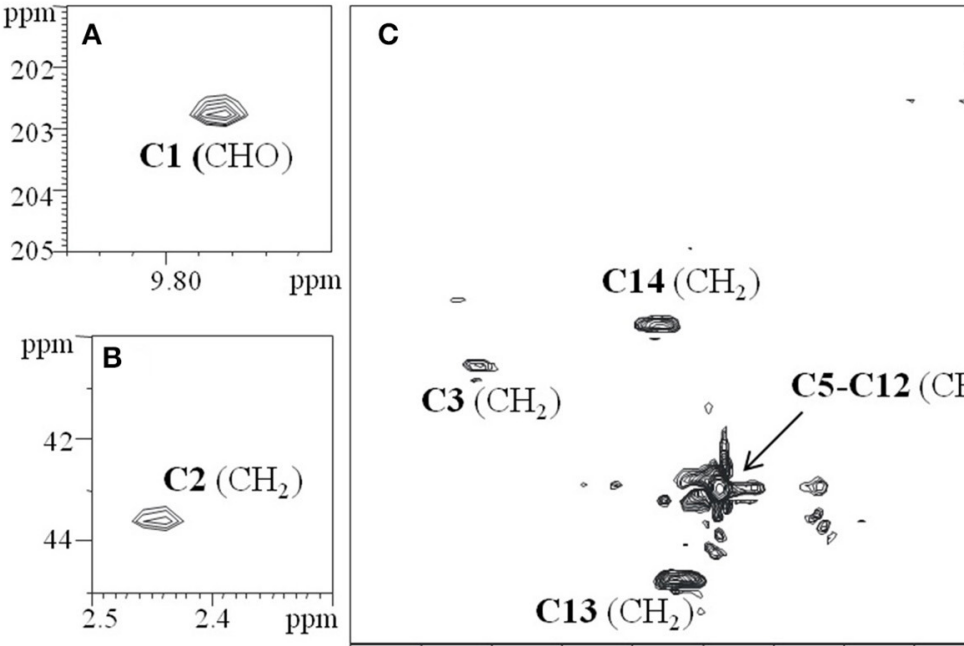
S. epidermidis biofilm, that was found to be active (Figure 3A). Consequently, commercial pentadecanal was used for all further experiments.

\section{Anti-Biofilm Activity and Specificity of Pentadecanal on S. epidermidis Biofilm}

Once the structure of the anti-biofilm molecule had been identified, the first step was to confirm the absence of any antimicrobial activity of pentadecanal on the staphylococcal reference strains by determining the minimum inhibitory concentration. The data obtained indicated no bacteriostatic and no bactericidal activity within the range of concentrations explored (the highest concentration explored was $1,6 \mathrm{mg} / \mathrm{ml}$ ). Preliminary experiments were also carried out to assess the effects of the molecule on the planktonic growth rate of S. epidermidis. The results obtained showed that pentadecanal did not affect the staphylococcal duplication rate. Bacterial growth curves were superimposable both in the presence and absence of pentadecanal (data not shown).

The pentadecanal activity on biofilm formation was assessed on two different $S$. epidermidis strains (Figure 3A), according to previously reported data that demonstrated the cell-free

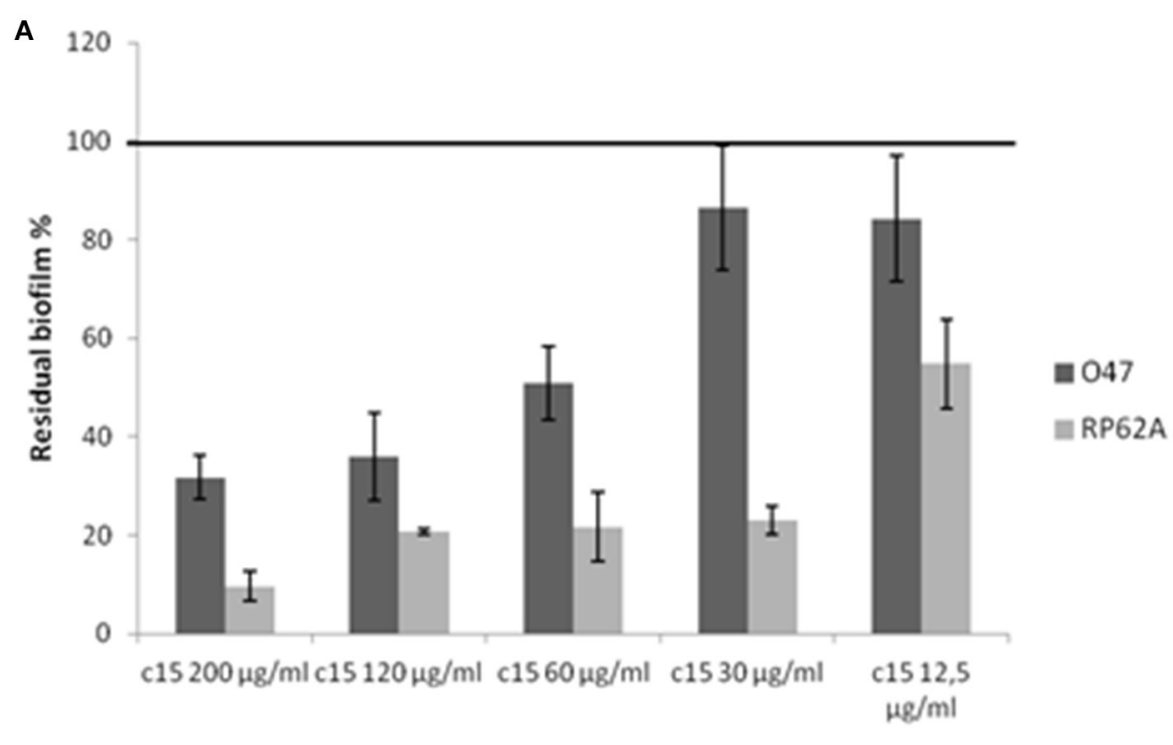

B

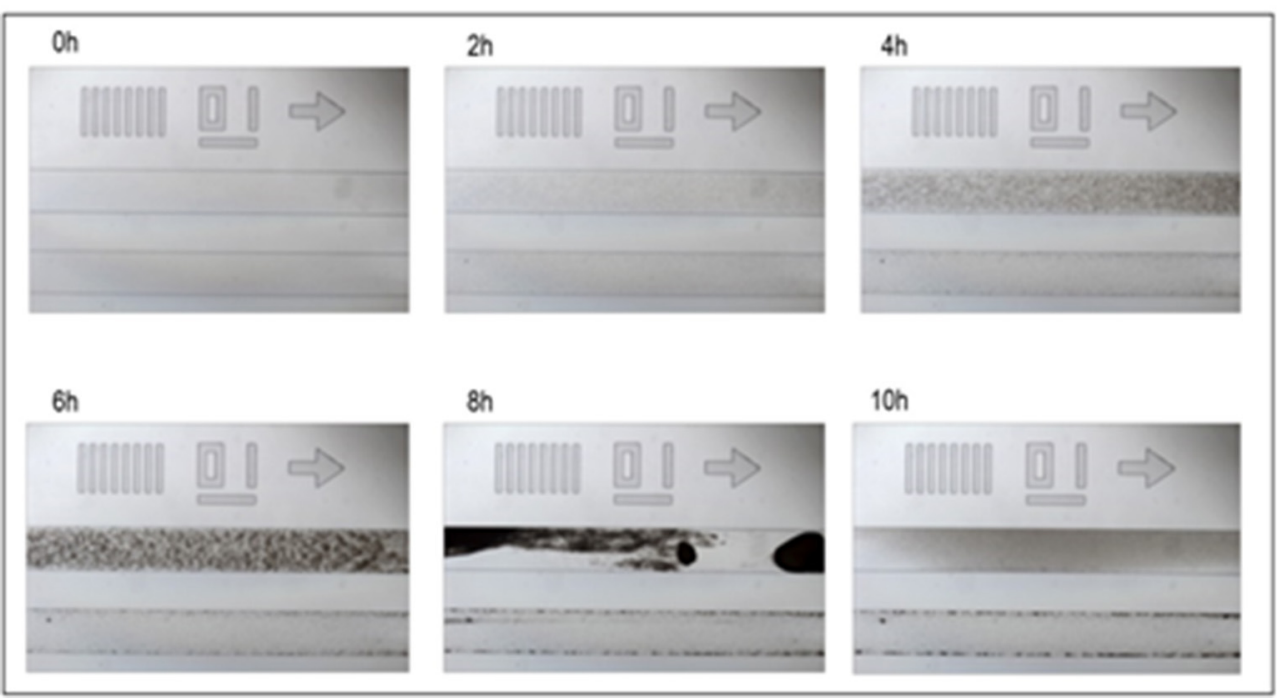

FIGURE 3 | Pentadecanal anti-biofilm activity on S. epidermidis biofilm formation. (A) The effect of pentadecanal at different concentrations on biofilm formation of S. epidermidis O-47 and S. epidermidis RP62A. The data are reported as percentages of residual biofilm. Each data point is composed of three independent samples. (B) Biofilm formation of S. epidermidis O-47 in a BioFlux system in the presence of pentadecanal. Each image contains two channels: the bottom channel is the pentadecanal-treated sample and the top channel is the control. Bright-field microscopic images were collected at 1-min intervals. The images presented were taken from the complete set of 720 images taken at 40x magnification. 
supernatant activity of $P$. haloplanktisTAC125 against the S. epidermidis O-47 and S. epidermidis RP62A biofilms (Papa et al., 2013b). As expected, the pentadecanal inhibitory effect was clearly dose-dependent with an efficacy higher than $50 \%$ already at $60 \mu \mathrm{g} / \mathrm{ml}$ (50.9\% residual biofilm for S. epidermidis O-47 and $21.8 \%$ for $S$. epidermidis RP62A, respectively). All previously described experiments were performed in BHI medium. The pentadecanal anti-biofilm activity was also tested in TSB medium to verify its effectiveness in function of growth medium composition. The results demonstrated that this latter did not influence the anti-biofilm ability of pentadecanal (untreated bacteria $0.761 \pm 0.137$; treated bacteria $0.152 \pm 0.020$ ).

The pentadecanal anti-biofilm efficacy was also tested in dynamic conditions with the BioFlux system, a microfluidic device that precisely controls the flow of growth medium between two interconnected wells of a microtiter plate. By positioning the channel connecting the two wells over a window accessible for viewing by microscopy, biofilm growth can be monitored in a time-course assay in which images are collected at 1-min intervals. In Figure 3B selected images of the BioFlux analysis are reported, showing the biofilm development of $S$. epidermidis O-47 at different times, in the absence or in the presence of pentadecanal (top and bottom lanes of each panel, respectively). The bacteria were seeded in both channels visible in each frame and after $30 \mathrm{~min}$ the flow was applied. The images collected showed an initial rapid growth of the bacteria, resulting in a confluent "lawn" of cells that was followed by a period of detachment. Pentadecanal clearly impaired the biofilm formation confirming the results obtained in the static system.

The pentadecanal effect on $S$. epidermidis O-47 biofilm was further investigated by confocal laser scanning microscopy (Figures 4A-D). CLSM was used to analyse the biofilm structure and viability, as shown in Figures 4B,D. In the presence of pentadecanal, as expected, the biofilm thickness decreased significantly with a notable alteration in the architecture. This effect was evident both on S. epidermidis O-47 biofilm and on $S$. epidermidis RP62A biofilm. Moreover, the viability of cells encapsulated in the biofilm in the presence and in the absence of pentadecanal was evaluated by live/dead staining. As shown in Figure 4C, cells exposed to pentadecanal were alive (green indicates viable cells while red indicates dead cells) confirming that the long-chain fatty aldehyde had no bactericidal activity on the S. epidermidis cells living in the biofilm.

In order to investigate the chemical features (the length of the chain and the functional group nature) that are essential for the anti-biofilm activity, the effect of similar aldehydes and corresponding alcohols, characterized by different lengths of the aliphatic chain in the range from C-14 to C-20, were analyzed and the results are summarized in Figure 5. The data are reported as percentages of residual biofilm after the treatment in comparison

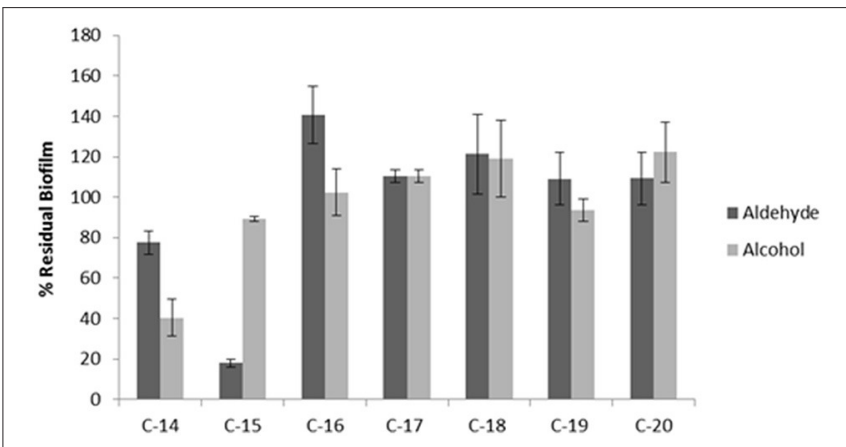

FIGURE 5 | Anti-biofilm activity of different aldehydes and alcohols on S. epidermidis $\mathbf{0 - 4 7}$. The anti-biofilm activity of different long-chain aldehydes and alcohols on S. epidermidis O-47. The data are reported as percentages of residual biofilm. Each data point is composed of four independent samples.
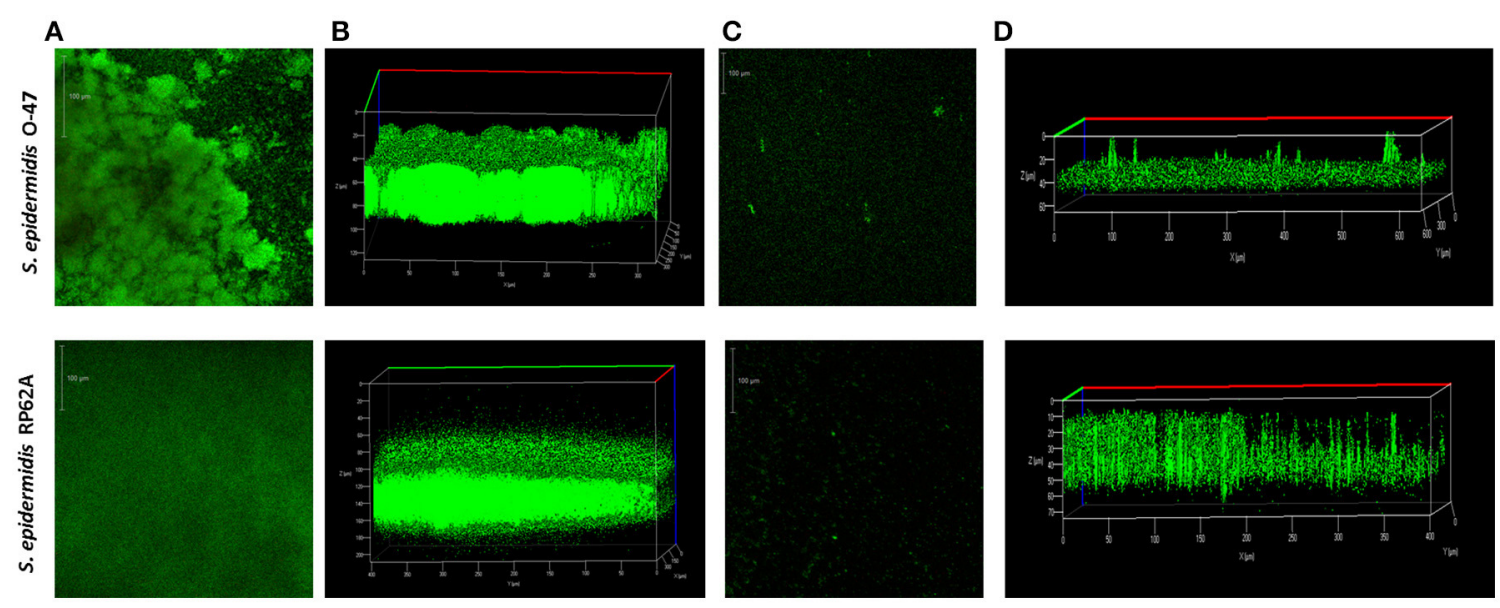

FIGURE 4 | CLSM of S. epidermidis 0-47 and S. epidermidis RP62A biofilms in the presence and absence of pentadecanal. (A) CLSM of S. epidermidis O-47 and S. epidermidis RP62A biofilms in BHI medium without pentadecanal and (C) with pentadecanal $100 \mu \mathrm{g} / \mathrm{ml}$. The bacteria were grown in chamber slides for $20 \mathrm{~h}$ and then stained with LIVE/DEAD reagents. The green fluorescence (SYTO9) indicates viable cells PI and the red fluorescence (PI) indicates dead cells. (B) Z-stack analysis of S. epidermidis O-47 and RP62A biofilms without pentadecanal. (D) Z-stack analysis of S. epidermidis O-47 and RP62A biofilms treated with 100 $\mu \mathrm{g} / \mathrm{ml}$ pentadecanal. 
with the untreated biofilm of S. epidermidis O-47. Our results clearly showed that, except for a partial reduction observed after treatment with the alcohol at the $\mathrm{C}-14$ chain, the anti-biofilm activity was limited to pentadecanal.

\section{Pentadecanal As an Al-2 Signaling Molecule in the LuxS/AI-2 QS System}

To test whether the anti-biofilm activity displayed by pentadecanal is correlated with a modulation/activation of the AI-2 quorum sensing system, the effect of pentadecanal was investigated on a $V$. harveyi $\mathrm{BB} 170$ strain (luxN::tn5Kan), a mutant sensor strain that responds only to AI-2 autoinducers. The $V$. harveyi BB170 bioluminescence was monitored (Figure 6) after adding two different concentrations of pentadecanal. At a low concentration $(12.5 \mu \mathrm{g} / \mathrm{ml})$ the pentadecanal was able to increase the $V$. harveyi bioluminescence (Figure 6), indicating that the $V$. harveyi LuxS/AI-2 QS system responds to pentadecanal. At a higher concentration $(200 \mu \mathrm{g} / \mathrm{ml})$ pentadecanal reduced the bioluminescence (Figure 6). In this condition, pentadecanal probably inhibits the $V$. harveyi luciferase. Since the $V$. harveyi luciferase physiological substrate is tetradecanal (Ulitzur and Hastings, 1979), the bioluminescence in $V$. harveyi BB170 was also monitored after adding two different concentrations of tetradecanal (Figure S1). As expected, a higher concentration $(200 \mu \mathrm{g} / \mathrm{ml})$ of tetradecanal reduced the $V$. harveyi bioluminescence due to the luciferase substrate inhibition, at low concentrations tetradecanal had no effect on the $V$. harveyi bioluminescence (Figure S1).

\section{The Role of Pentadecanal in the P. haloplanktisTAC125 Physiology}

To assess the role of pentadecanal in the P. haloplanktisTAC125 physiology, we investigated the conditions necessary for pentadecanal production and the possible synthetic pathway involved.

The in silico analysis of the $P$. haloplanktisTAC125 genome (EMBL under accession nos. CR954246 and CR954247). did not allow us to identify the genes coding for enzymes known to be able to synthesize fatty aldehydes, like fatty acid reductase (EC 1.2.1.50 (Gahan, 2012) or fatty acid peroxidase (EC1.11.1.3, Martin and Stumpf, 1959). However, the analysis allowed the identification of a gene (PSHAb0219) coding for an aldehyde dehydrogenase B (EC1.2.1.3), an enzyme annotated as a reversible aldehyde oxidoreductase $\mathrm{NAD}(\mathrm{P})^{+}$-dependent with a wide specificity (http://www.genoscope.cns.fr/agc/ microscope/mage/viewer.php). The aldehyde oxidoreductase, in specific conditions, could be able to produce pentadecanal by reducing the corresponding fatty acid. To assess if this was a correct hypothesis, we first investigated the presence of the pentadecanoic acid in P. haloplanktis TAC125 cells. A GC-MS analysis of fatty acid methyl esters from $P$. haloplanktis TAC 125 cells, grown either in sessile or in planktonic conditions, revealed the presence of pentadecanoic acid (Figure S2).

Once the presence of the possible substrate had been verified, both in sessile and in planktonic conditions, a RT PCR analysis of the PSHAb0219 gene was performed to assess if the gene transcription is growth condition-dependent. P. haloplanktis TAC125 cells were cultured in planktonic or in sessile conditions and total RNA was extracted from the two different samples. The PSHAb0219 gene amplification was obtained with specific oligonucleotide pairs (see Material and Methods Section). The results of the RT-PCR experiments (Figure S3) showed that a PSHAb0219 gene transcription was clearly detected in the RNA samples extracted from $P$. haloplanktis TAC125 cells grown in both conditions. Several experiments were then attempted to delete the PSHAb0219 gene by using genetic tools for the creation of insertion/deletion

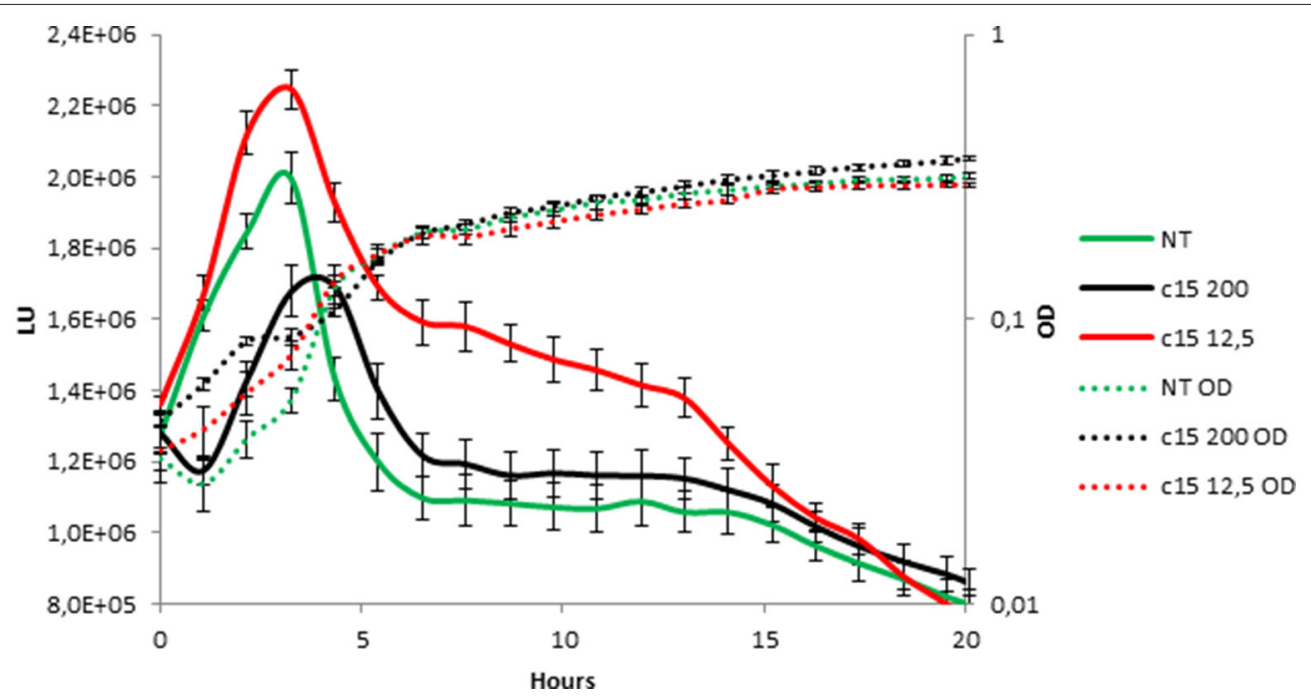

FIGURE 6 | V. harveyi LuxS/AI-2 QS system responds to the presence of pentadecanal. Bioluminescence (solid lines) and growth curves (dotted lines) of the $V$. harveyi BB170 strain incubated for $20 \mathrm{~h}$ in the presence of only the AB medium (green lines), $12.5 \mu \mathrm{g} / \mathrm{mL}$ pentadecanal (black lines) and $200 \mu \mathrm{g} / \mathrm{mL}$ pentadecanal concentrations (red lines). 
mutants already available (Parrilli et al., 2010). However, these experiments were not successful probably due to the fact that PSHAb0219, in tested conditions, is likely to be an essential gene.

Since the pentadecanoic acid was produced in both sessile and planktonic conditions and the PSHAb0219 gene was transcribed in both settings, a possible explanation of the presence of pentadecanal only in the supernatant of the sessile form could be that the aldehyde oxidoreductase catalyses the reduction of the acid to aldehyde only in the biofilm condition. In particular, in oxygen limitation, a condition occurring in biofilm (Wessel et al., 2014), bacterial cells could reduce the fatty acids in order to obtain the oxidized cofactor $\left(\mathrm{NAD}(\mathrm{P})^{+}\right)$necessary for cell metabolism. To verify this hypothesis $P$. haloplanktis TAC125 cells were grown, in an automatic bioreactor, at $4^{\circ} \mathrm{C}$ in microaerobiosis under agitation, a condition where the measured oxygen pressure is kept constantly below 5\% saturation (measured oxygen pressure $<5 \%$ )(see Materials and Methods Section). The anti-biofilm activity of the cell-free supernatant of $P$. haloplanktis TAC125 grown in microaerobiosis (SN-M) was tested on $S$. epidermidis biofilm. The SN-M proved to be able to inhibit the $S$. epidermidis $\mathrm{O}-47$ biofilm formation (Figure 7), thus indicating that the pentadecanal production occurs also in microaerobiosis. This experiment demonstrates that oxygen availability is a crucial parameter for pentadecanal synthesis and proves to be the main difference between sessile and planktonic conditions, so determining the anti-biofilm production.

Although pentadecanal could be not defined as a strictly biofilm-specific metabolite, its effect on $P$. haloplanktis TAC125 biofilm formation and development was tested. The aldehyde presence proved to have no effect (Figure S4) on the Antarctic bacterium biofilm development. Moreover, pentadecanal proved to be inactive against the mature biofilm of $P$. haloplanktis TAC125 (data not shown).

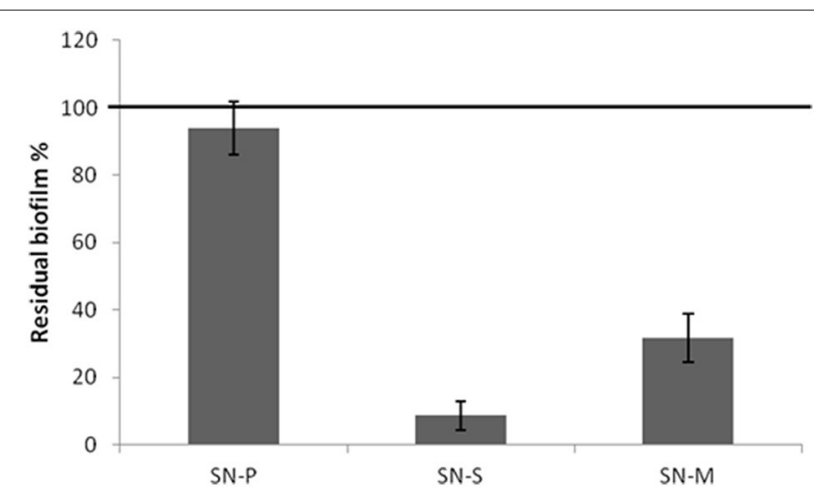

FIGURE 7 | The cell-free supernatant of $P$. haloplanktis TAC125 grown in microaerobiosis has an anti-biofilm activity $\mathrm{S}$. epidermidis $\mathrm{O}-47$ biofilm formation after incubation with P. haloplanktis TAC125 cell-free supernatants obtained from sessile (SN-S) and planktonic growths (SN-P) and from microaerobiosis growth (SN-M). The data are reported as percentages of residual biofilm. Each data point represents the mean \pm SD of at least three independent samples.

\section{DISCUSSION}

The emergence of Staphylococcus epidermidis as an opportunistic pathogen is closely related to the biofilm forming capability of this bacterial species, especially during the biofilm-associated infection of indwelling medical devices. The increasing use of implanted medical devices heightens the importance of $S$. epidermidis as a human pathogen. Our research has been aimed at discovering anti-biofilm molecules from natural sources, such as marine microbiota, since we are convinced that this approach will lead to the discovery of novel and unforeseen compounds. Cold-adapted marine bacteria represent a still underexploited source of biodiversity able to synthesize a broad range of bioactive compounds, including anti-biofilm molecules. Previous results (Papa et al., 2013b; Parrilli et al., 2015) have demonstrated that the culture supernatant of Antarctic marine bacterium P. haloplanktis TAC125 impairs the formation of S. epidermidis biofilm.

In this paper we applied a P. haloplanktis TAC125 biofilm cultivation strategy in automatic bioreactor (Parrilli et al., 2016) and an efficient activity-guided purification protocol to produce and purify the anti-biofilm molecule. The structure of the antibiofilm molecule, obtained by NMR and mass spectrometry, corresponds to pentadecanal, a long-chain fatty aldehyde.

The pentadecanal activity on biofilm formation was confirmed on two different $S$. epidermidis strains in static condition and, by the BioFlux system, also in dynamic conditions. BioFlux can reproduce environmental or physiological conditions by precisely controlling shear flow, bridging the gap between in vitro and in vivo assays. In Bioflux conditions bacteria progress through a series of developmental steps, ultimately forming a multicellular structure containing differentiated cell populations. The observation of the growing biofilm at various time-points throughout this process provides a glimpse of the temporal changes that occur. Flow biofilm is more closely related to natural biofilms and can differ from static biofilms in terms of the hydrodynamic influences on cell signaling. The finding that pentadecanal is active also in dynamic conditions demonstrates that this molecule shows great promise in terms of its use in vivo systems.

It is interesting to note that most of the known anti-biofilm molecules also display an antibacterial activity (bactericidal or bacteriostatic). In contrast, pentadecanal lacks any antibacterial activity against free-living bacteria and against bacteria entrapped in biofilm matrix. Indeed as demonstrated by CLSM analysis the presence of pentadecanal reduces the biofilm thickness without affecting the viability of $S$. epidermidis cells living in the biofilm. Therefore, pentadecanal targets the adhesive properties without affecting the bacterial viability, a type of behavior which should prevent the development of escape mutants. This property makes pentadecanal particularly interesting and suggests its possible use in combination with conventional antibiotic therapy.

The specificity of pentadecanal action on S. epidermidis biofilm has been demonstrated by testing chemical analogs differing either in the length of the aliphatic chain or in their functional group properties. The results reported demonstrate that both the length of the aliphatic chain and the functional 
group properties are essential for the pentadecanal activity against $S$. epidermidis biofilm.

With regard to the anti-biofilm mode of action, several observations prompted us to explore the idea that the action of pentadecanal on $S$. epidermidis biofilm could be related to its possible interference in the quorum sensing system. The most important and best-characterized quorum-sensing system in staphylococci is the accessory gene regulator (agr) system. Remarkably, the $P$. haloplanktis TAC125 anti-biofilm molecule is active on the clinical isolate $\mathrm{O}-47$, which is a naturally occurring agr mutant (Vuong et al., 2003); therefore a possible effect mediated by quorum sensing should be directed at the LuxS/AI2 quorum sensing system (Xu et al., 2006; Li et al., 2008), the second quorum sensing system present in S. epidermidis. The role of the LuxS/AI-2 QS system in S. epidermidis biofilm regulation is under debate. $\mathrm{Xu}$ and co-authors have suggested that the autoinducer 2 (AI-2) negatively regulates the expression of the ica gene [ica operon encodes enzymes responsible for the production of polysaccharide intercellular adhesion PIA (Otto, 2008)] at the transcriptional level, reducing the PIA synthesis and biofilm formation (Xu et al., 2006). In contrast, a recent paper of Xue et al. (2015) has reported that AI-2 increased biofilm formation in S. epidermidis RP62A by enhancing the transcription of the ica operon. Moreover, in a paper (Li et al., 2008) concerning AI-2 dependent gene regulation in S. epidermidis, it has been demonstrated that AI-2 controls also the levels of phenolsoluble modulins (PSMs), key molecules able to lead to biofilm detachment of $S$. epidermidis biofilm in vivo e in vitro (Otto, 2013). In any case, the crucial role of the LuxS/AI-2 QS system in S. epidermidis biofilm formation is evident, while its role in Staphylococcus aureus biofilm formation is less clear; indeed, several studies suggest that the LuxS/AI-2 quorum sensing system has no role in $S$. aureus biofilm formation (Doherty et al., 2006; Cluzel et al., 2010). The differences in the involvement of AI-2 molecules in biofilm formation between $S$. aureus and $S$. epidermidis could explain why the P. haloplanktis TAC125 antibiofilm compound is effective against the $S$. epidermidis biofilm but is not able to inhibit the S. aureus biofilm (Papa et al., 2013b). To test whether the anti-biofilm activity displayed by pentadecanal is correlated with a modulation/activation of the AI-2 quorum sensing system, the effect of pentadecanal was investigated on a $V$. harveyi mutant sensor strain that responds only to the AI-2 autoinducers.

The results reported demonstrate that at low concentration the pentadecanal is able to increase the $V$. harveyi bioluminescence, indicating that the $V$. harveyi LuxS/AI-2 QS system responds to pentadecanal, at a higher concentration it reduces the bioluminescence. In this condition, pentadecanal probably inhibits the $V$. harveyi luciferase. The key reaction in bacterial bioluminescence is the oxidation of the luciferasecatalyzed $\mathrm{FMNH}_{2}$ and the long chain aliphatic aldehyde (Byers et al., 1988), and, consequently, the bacterial luciferase, that can utilize fatty aldehydes of varying chain lengths, is inhibited by the high levels of aldehydes (Francisco et al., 1993). Therefore, it is reasonable to assume that also pentadecanal could be an inhibitor of the $V$. harveyi luciferase. Since the $V$. harveyi luciferase physiological substrate is tetradecanal (Ulitzur and
Hastings, 1979), the bioluminescence in $V$. harveyi mutant was also monitored in presence of tetradecanal, and, as expected, a higher concentration reduced the $V$. harveyi bioluminescence due to the luciferase substrate inhibition. It is interesting to note that at low concentrations tetradecanal had no effect on the $V$. harveyi bioluminescence.

The results reported demonstrate that the V. harveyi LuxS/AI2 QS system responds to pentadecanal. These data suggest that the pentadecanal anti-biofilm activity in S. epidermidis could be due to an interference in the AI-2 quorum sensing system. Although several studies will be necessary to clarify the molecular details of the pentadecanal action on S. epidermidis biofilm formation, this is the first report on the action of a long-chain fatty aldehyde as an anti-biofilm molecule that works as a signaling molecule in an AI-2 QS system. Further investigations will be necessary to clarify if this modulation occurs also in the $S$. epidermidis AI-2 quorum sensing system.

Besides the characterization of pentadecanal anti-biofilm activity on $S$. epidermidis biofilm, we have investigated the metabolic role and synthesis of this long fatty compound in the Antarctic source strain. In a previous work we examined the anti-biofilm activity of cell-free supernatants obtained from the $P$. haloplanktis TAC125 grown in sessile or in planktonic conditions on different staphylococci (Papa et al., 2013b). Our results demonstrated that only when $P$. haloplanktisTAC125 is grown in sessile condition the cell-free supernatant inhibit the biofilm formation of $S$. epidermidis. This latter result was explained by considering that biofilm specific environmental conditions may induce a profound genetic and metabolic rewiring allowing the production of biofilm-specific metabolites (Beloin and Ghigo, 2005). To evaluate if pentadecanal can be considered a biofilm-specific metabolite, we investigated the conditions necessary for pentadecanal production and the possible synthetic pathway involved. The results reported strongly suggest that a $P$. haloplanktisTAC125 reversible aldehyde oxidoreductase $\mathrm{NAD}(\mathrm{P})+$-dependent catalyses, in oxygen limitation, the reduction of the pentadecaonic acid to aldehyde to obtain the oxidized cofactor $(\mathrm{NAD}(\mathrm{P})+)$ necessary for cell metabolism. Therefore, the pentadecanal production occurs in the biofilm condition and in microaerobiosis due to the reduced oxygen availability that characterizes these two growth conditions. The oxygen availability results a crucial parameter for pentadecanal synthesis and proves to be the main difference between sessile and planktonic conditions, so determining the anti-biofilm production. Moreover, the results described in this paper indicate that the long-chain fatty aldehyde is not involved in the control/modulation of the Antarctic bacterium biofilm development.

In conclusion, this is the first report both on the action of a long chain fatty aldehyde as an anti-biofilm molecule and on an aldehyde working as a signaling molecule in the AI-2 QS system. We believe that this paper endorses the potential of cold-adapted marine bacteria as a source of bioactive compounds of interest, and contributes to the development of innovative approaches for the prevention and treatment of S. epidermidis biofilm-associated infections. 


\section{AUTHOR CONTRIBUTIONS}

AC: Performed the experiments, suggested critical parameters in design of experiments and co-wrote the paper. RP: Performed the experiments, suggested critical parameters in design of experiments and co-wrote the paper. AR: Performed the experiments and co-wrote the paper. FS, MZ and MT: Performed the experiments. LS: Provided advice in performance of experiments and edited paper. GM: Provided advice in performance of experiments and co-wrote paper. MC: Suggested critical parameters in design of experiments and co-wrote paper. MLT: Suggested critical parameters in design of experiments and co-wrote paper. MA: Provided advice in performance of experiments and

\section{REFERENCES}

Arciola, C. R., Hänsch, G. M., Visai, L., Testoni, F., Maurer, S., Campoccia, D., et al. (2012). Interactions of staphylococci with osteoblasts and phagocytes in the pathogenesis of implant-associated osteomyelitis. Int. J. Artif. Organs. 35, 713-726. doi: 10.5301/ijao.5000158

Artini, M., Papa, R., Scoarughi, G. L., Galano, E., Barbato, G., Pucci, P., et al. (2013). Comparison of the action of different proteases on virulence properties related to the staphylococcal surface. J. Appl. Microbiol. 114, 266-277. doi: $10.1111 /$ jam. 12038

Beloin, C., and Ghigo, J. M. (2005). Finding gene-expression patterns in bacterial biofilms. Trends Microbiol. 13, 16-19. doi: 10.1016/j.tim.2004.11.008

Byers, D. M., Bognar, A., and Meighen, E. A. (1988). Differential regulation of enzyme activities involved in aldehyde metabolism in the luminescent bacterium Vibrio harveyi. J. Bacteriol. 170, 967-971. doi: 10.1128/jb.170.2.967-971.1988

Cluzel, M. E., Zanella-Cléon, I., Cozzone, A. J., Fütterer, K., Duclos, B., and Molle, V. (2010). The Staphylococcus aureus autoinducer-2 synthase LuxS is regulated by Ser/Thr phosphorylation. J. Bacteriol. 192, 6295-6301. doi: 10.1128/JB.00853-10

Dohar, J. E., Hebda, P. A., Veeh, R., Awad, M., Costerton, J. W., Hayes, J., et al. (2009). Mucosal biofilm formation on middle-ear mucosa in a nonhuman primate model of chronic suppurative otitis media. Laryngoscope 115, 1469-1472. doi: 10.1097/01.mlg.0000172036. 82897.d4

Doherty, N., Holden, M. T. G., Qazi, S. N., Williams, P., and Winzer, K. (2006). Functional analysis of luxS in Staphylococcus aureus reveals a role in metabolism but not quorum sensing. J. Bacteriol. 188, 2885-2897. doi: 10.1128/JB.188.8.2885-2897.2006

Epstein, A. K., Wong, T. S., Belisle, R. A., Boggs, E. M., and Aizenberg, J. (2012). Liquid-infused structured surfaces with exceptional antibiofouling performance. Proc. Natl. Acad. Sci. U.S.A. 109, 13182-13187. doi: 10.1073/pnas.1201973109

Francisco, W. A., Abu-Soud, H. M., Baldwin, T. O., and Raushel, F. M. (1993). Interaction of bacterial luciferase with aldehyde substrates and inhibitors. J. Biol. Chem. 268, 24734-24741.

Gahan, C. G. (2012). The bacterial lux reporter system: applications in bacterial localisation studies. Curr. Gene Ther. 12, 12-19. doi: 10.2174/156652312799789244

Giuliani, M., Parrilli, E., Pezzella, C., Rippa, V., Duilio, A., Marino, G., et al. (2012). A novel strategy for the construction of genomic mutants of the Antarctic bacterium Pseudoalteromonas haloplanktis TAC125. Methods Mol. Biol. 824, 219-233. doi: 10.1007/978-1-61779-433-9_11

Heilmann, C., Gerke, C., Perdreau-Remington, F., and Götz, F. (1996). Characterization of Tn917 insertion mutants of Staphylococcus epidermidis affected in biofilm formation. Infect. Immun. 64, 277-282.

Iebba, V., Totino, V., Santangelo, F., Gagliardi, A., Ciotoli, L., Virga, A., et al. (2014). Bdellovibrio bacteriovorus directly attacks Pseudomonas aeruginosa edited paper. EP: Designed the experiments, provided advice in performance of experiments and wrote the paper.

\section{FUNDING}

This work was supported by Programma Nazionale di Ricerche in Antartide 2013/B1.04 Tutino.

\section{SUPPLEMENTARY MATERIAL}

The Supplementary Material for this article can be found online at: http://journal.frontiersin.org/article/10.3389/fcimb. 2017.00046/full\#supplementary-material

and Staphylococcus aureus cystic fibrosis isolates. Front. Microbiol. 5:280. doi: 10.3389/fmicb.2014.00280

Kaplan, J. B. (2010). Biofilm dispersal: mechanisms, clinical implications and potential therapeutic uses. J. Dent. Res. 89, 205-218. doi: $10.1177 / 0022034509359403$

Kiran, G. S., Sabarathnam, B., and Selvin, J. (2010). Biofilm disruption potential of a glycolipid biosurfactant from marine Brevibacterium casei. FEMS Immunol. Med. Microbiol. 59, 432-438. doi: 10.1111/j.1574-695X.2010.00698.x

Klein, G. L., Soum-Soutéra, E., Guede, Z., Bazire, A., Compère, C., and Dufour, A. (2011). The anti-biofilm activity secreted by a marine Pseudoalteromonas strain. Biofouling 27, 931-940. doi: 10.1080/08927014.2011.611878

Li, M., Villaruz, A. E., Vadyvaloo, V., Sturdevant, D. E., and Otto, M. (2008). AI-2dependent gene regulation in Staphylococcus epidermidis. BMC Microbiol. 8:4. doi: 10.1186/1471-2180-8-4

Martin, R. O., and Stumpf, P. K. (1959). Fat metabolism in higher plants. XII. alpha-Oxidation of long chain fatty acids. J. Biol. Chem. 234, 2548-2554.

Médigue, C., Krin, E., Pascal, G., Barbe, V., Bernsel, A., Bertin, P. N., et al. (2005). Coping with cold: the genome of the versatile marine Antarctica bacterium Pseudoalteromonas haloplanktis TAC125. Genome Res. 15, 1325-1335. doi: 10.1101/gr.4126905

Miller, R. A., and Hoerrner, R. S. (2003). Iodine as a chemoselective reoxidant of TEMPO: application to the oxidation of alcohols to aldehydes and ketones. Org. Lett. 5, 285-287. doi: 10.1021/ol0272444

Ni, N., Li, M., Wang, J., and Wang, B. (2009). Inhibitors and antagonists of bacterial quorum sensing. Med. Res. Rev. 29, 65-124. doi: 10.1002/med.20145

Otto, M. (2008). Staphylococcal biofilm. Curr. Top. Microb. Immunol. 322, 207-228. doi: 10.1007/978-3-540-75418-3_10

Otto, M. (2013). Staphylococcal infections: mechanisms of biofilm maturation and detachment as critical determinants of pathogenicity. Annu. Rev. Med. 64, 175-188. doi: 10.1146/annurev-med-042711-140023

Papa, R., Artini, M., Cellini, A., Tilotta, M., Galano, E., Pucci, P., et al. (2013a). A new anti-infective strategy to reduce the spreading of antibiotic resistance by the action on adhesion-mediated virulence factors in Staphylococcus aureus. Microb. Pathog. 63, 44-53. doi: 10.1016/j.micpath.2013.05.003

Papa, R., Parrilli, E., Sannino, F., Barbato, G., Tutino, M. L., Artini, M., et al. (2013b). Anti-biofilm activity of the Antarctic marine bacterium Pseudoalteromonas haloplanktis TAC125. Res. Microbiol. 164, 450-456. doi: 10.1016/j.resmic.2013.01.010

Papa, R., Selan, L., Parrilli, E., Tilotta, M., and Sannino, F. (2015). Anti-biofilm activities from marine cold adapted bacteria against Staphylococci and Pseudomonas aeruginosa. Front. Microbiol. 4:1333. doi: 10.3389/fmicb.2015.01333

Parrilli, E., Giuliani, M., Pezzella, C., Danchin, A., Marino, G., and Tutino, M. L. (2010). PssA is required for $\alpha$-amylase secretion in Antarctic Pseudoalteromonas haloplanktis. Microbiology 156, 211-219. doi: 10.1099/mic.0.032342-0

Parrilli, E., Papa, R., Carillo, S., Tilotta, M., Casillo, A., Sannino, F., et al. (2015). Anti-biofilm activity of Pseudoalteromonas haloplanktis TAC125 against 
Staphylococcus epidermidis biofilm: evidence of a signal molecule involvement? Int. J. Immunopathol. Pharmacol. 28, 104-113. doi: 10.1177/0394632015 572751

Parrilli, E., Ricciardelli, A., Casillo, A., Sannino, F., Papa, R., Tilotta, M., et al. (2016). Large-scale biofilm cultivation of Antarctic bacterium Pseudoalteromonas haloplanktis TAC125 for physiologic studies and drug discovery. Extremophiles 20, 227-234. doi: 10.1007/s00792-016-0813-2

Qin, Z., Yang, L., Qu, D., Molin, S., and Tolker-Nielsen, T. (2009). Pseudomonas aeruginosa extracellular products inhibit staphylococcal growth, and disrupt established biofilms produced by Staphylococcus epidermidis. Microbiology 155, 2148-2156. doi: 10.1099/mic.0.028001-0

Rippa, V., Papa, R., Giuliani, M., Pezzella, C., Parrilli, E., Tutino, M. L., et al. (2012). Regulated recombinant protein production in the Antarctic bacterium Pseudoalteromonas haloplanktis TAC125. Methods Mol. Biol. 824, 203-218. doi: 10.1007/978-1-61779-433-9_10

Sun, J., Kan, F., Liu, P., He, S., Mou, H., Xue, C., et al. (2015). Screening of microorganisms from deep-sea mud for Antarctic krill (Euphausia superba) fermentation and evaluation of the bioactive compounds. Appl. Biochem. Biotechnol. 175, 1664-1677. doi: 10.1007/s12010-0141403-3

Taga, M. E. (2005). Methods for analysis of bacterial autoinducer-2 production. Curr. Protoc. Microbiol. Chapter 1:Unit 1C.1. doi: 10.1002/978047172 9259.mc01c01s00

Ulitzur, S., and Hastings, J. W. (1979). Evidence for tetradecanal as the natural aldehyde in bacterial bioluminescence. Proc. Natl. Acad. Sci. U.S.A. 76, 265-267. doi: $10.1073 /$ pnas.76.1.265

Valle, J., Da Re, S., Henry, N., Fontaine, T., Balestrino D., Latour-Lambert, P., et al. (2006). Broad-spectrum biofilm inhibition by a secreted bacterial polysaccharide. Proc. Natl. Acad. Sci. U.S.A. 103, 12558-12563. doi: $10.1073 /$ pnas. 0605399103

Vuong, C., Gerke, C., Somerville, G. A., Fischer, E. R., and Otto, M. (2003). Quorum-sensing control of biofilm factors in Staphylococcus epidermidis. J. Infect. Dis. 188, 706-718. doi: 10.1086/377239

Wessel, A. K., Arshad, T. A., Fitzpatrick, M., Connell, J. L., Bonnecaze, R. M., Shear, J. B., et al. (2014). Oxygen limitation within a bacterial aggregate. MBio 15:e00992. doi: 10.1128/mBio.00992-14

Xu, L., Li, H., Vuong, C., Vadyvaloo, V., Wang, J., Yao, Y., et al. (2006). Role of the luxS quorum-sensing system in biofilm formation and virulence of Staphylococcus epidermidis. Infect. Immun. 74, 488-496. doi: 10.1128/IAI.74.1.488-496.2006

Xue, T., Ni, J., Shang, F., Chen, X., and Zhang, M. (2015). Autoinducer2 increases biofilm formation via an ica- and $b h p$-dependent manner in Staphylococcus epidermidis RP62A. Microbes Infect. 17, 345-352. doi: 10.1016/j.micinf.2015.01.003

Conflict of Interest Statement: The authors declare that the research was conducted in the absence of any commercial or financial relationships that could be construed as a potential conflict of interest.

Copyright (c) 2017 Casillo, Papa, Ricciardelli, Sannino, Ziaco, Tilotta, Selan, Marino, Corsaro, Tutino, Artini and Parrilli. This is an open-access article distributed under the terms of the Creative Commons Attribution License (CC BY). The use, distribution or reproduction in other forums is permitted, provided the original author(s) or licensor are credited and that the original publication in this journal is cited, in accordance with accepted academic practice. No use, distribution or reproduction is permitted which does not comply with these terms. 